\title{
A Pilot Study of Brief, Stepped Behavioral Activation for Primary Care Patients with Depressive Symptoms
}

\author{
Amber M. Gum ${ }^{1,2}$ (1) $\cdot$ Claudia Jensen ${ }^{1} \cdot$ Lawrence Schonfeld $^{1} \cdot$ Kyaien O. Conner $^{1} \cdot$ Lucy Guerra $^{2}$
}

Accepted: 11 February 2022 / Published online: 27 February 2022

(c) The Author(s), under exclusive licence to Springer Science+Business Media, LLC, part of Springer Nature 2022

\begin{abstract}
In a prior pilot study with primary care patients experiencing depressive symptoms, we observed positive outcomes for a behavioral activation protocol involving one visit and three calls. We aimed to pilot test a stepped version with flexible numbers of contacts. Fifteen primary care patients scoring 5-14 on the Patient Health Questionnaire-9 engaged in the three-tiered telephone-based intervention: (1) two calls (15-20 min each); (2) one 30-60 min encounter followed by two calls; and (3) one to six calls. Participants completed assessments at pre-treatment, post-treatment, and three months later. Participants improved from baseline to post-treatment and three months later for depressive symptoms, anxiety symptoms, and disability. Most participants ( 9 of 15) engaged in the first tier only, averaging less than one hour of contact, and reported benefits and high satisfaction. This intervention showed preliminary evidence of feasibility, acceptability, satisfaction, and benefits, warranting further pilot testing with primary care personnel.
\end{abstract}

Keywords Depression $\cdot$ Primary care $\cdot$ Behavioral activation $\cdot$ Adults $\cdot$ Brief intervention

\section{Introduction}

The impact of collaborative care for depression delivered in primary care settings is clear. Meta-analyses demonstrate that collaborative care improves depressive symptoms and other outcomes (e.g., anxiety symptoms, disability, and functioning) for primary care patients, compared to usual care (Bower et al., 2006; Woltmann et al., 2012). Not only is collaborative care effective, but it also improves access, engagement, and satisfaction with depression care services, and it saves money, compared to usual care (Katon et al., 2010; Zivin et al., 2016). Policy recommendations (Community Preventive Services Task Force, 2012) and payment models are being modified to support collaborative care activities (Miller et al., 2017; Press et al., 2017), further expanding their impact on primary care settings.

Amber M. Gum

ambergum@usf.edu

1 Department of Mental Health Law \& Policy, University of South Florida, 13301 Bruce B. Downs Blvd., MHC 2632A, Tampa, FL 33612, USA

2 Department of Internal Medicine, USF Health, University of South Florida, Tampa, USA
In collaborative care, a care manager engages the patient in screening and treatment planning that offers pharmacologic and/or behavioral interventions. The care manager collaborates with the patient's primary care physician and one or more behavioral health specialists to deliver care, monitor the patient's progress, and adjust treatment as needed. The care manager may be a social worker or behavioral health specialist but also may be a nurse or other healthcare provider without behavioral health specialization. Collaborative care models often utilize a stepped care approach, in which one treatment modality (e.g., behavioral intervention, medication) is initiated and then treatments are modified or augmented if the patient does not respond optimally to the initial course of treatment (e.g., changing dose or type of medication, adding medication, combining medication, and behavioral intervention; Katon \& Unützer, 2013). It is optimal for collaborative care programs to include on-site behavioral interventions because many patients desire these interventions and because collaborative care is particularly effective at matching patients' preferences for behavioral interventions (Gum et al., 2006). Also, behavioral interventions are an important treatment option to offer as part of collaborate care packages delivered in primary care settings, given that most patients have mild-moderate depressive symptoms that can be treated with behavioral interventions, 
thereby preventing their depression from worsening (Reynolds et al., 2012).

It can be challenging to learn, deliver, and sustain behavioral interventions in primary care settings (Alexopoulos et al., 2015; Cross et al., 2014; IOM, 2015), which is likely why some collaborative care programs have not offered behavioral interventions (Areán \& Gum, 2013). What is needed is an effective behavioral intervention that can be feasibly incorporated into collaborative care packages in primary care settings and delivered by care managers or other primary care personnel, who may not be behavioral health providers.

Behavioral activation, which is based on the premise that planning and engaging in more valued and enjoyable activities will enhance mood, is an excellent candidate to incorporate into collaborative care programs in primary care for several reasons. First, behavioral activation has a strong empirical evidence base across a variety of service settings and with diverse populations (Chartier \& Provencher, 2013; Dimidjian et al., 2011; Stein et al., 2021; Sturmey, 2009), including evidence of its effectiveness as a standalone behavioral intervention in primary care. Several studies have now demonstrated significant treatment effects for depression using 4-9-session behavioral activation protocols implemented in primary care settings (Funderburk et al., 2020, 2021; Gros \& Haren, 2011; Hopko et al., 2005; Trombello et al., 2017). A two-session behavioral activation protocol helped to reduce pain interference for primary care patients with chronic pain (Hooker et al., 2020). Some collaborative care programs for depression in primary care have incorporated components of behavioral activation to help patients plan pleasant activities along with medication management and other care management activities, with positive yet modest benefit (Gensichen et al., 2009; Richards et al., 2013). Second, behavioral activation is a core element of nearly all forms of behaviorally focused treatment (Alexopoulos \& Arean, 2014). Third, behavioral activation has been found to be more feasible to train social work and mental health providers to deliver than other behavioral interventions (Alexopoulos et al., 2015). Its feasibility likely derives from the straightforward nature of the rationale for behavioral activation, structured protocols, and patient materials. Further support for the feasibility of behavioral activation comes from one study of a very brief version of behavioral activation, in which depressed college students improved after one 90-min visit with a follow-up assessment 2 weeks later, with strong effect sizes compared to a no-treatment control group (Gawrysiak et al., 2009).

In a prior pilot study, our team modified this one-visit behavioral activation intervention (Gawrysiak et al., 2009) with 14 older primary care patients with mild-moderate depressive symptoms, with promising results (Gum et al., 2016). In one 90-min visit in the primary care clinic, participants identified life values and weekly behavior goals. Next, they were called three times over the next 4 weeks to monitor their progress toward the weekly goals and depressive symptoms. The outcomes were positive, with moderate to large improvements from pre-test to post-test in depressive symptoms, as well as anxiety symptoms and disability (which were impacted by collaborative care for depression in some prior studies, e.g., Woltmann et al., 2012; Unützer et al., 2002) and readiness to change (included as an indicator of participants' readiness to make behavioral change, an important mechanism of behavioral activation; Dimidjian et al., 2011). Although most participants were not depressed at the end of the intervention ( 8 of 14), four scored in the mildly depressed range and two remained in the moderately depressed range. This observation led us to ask whether we could obtain better outcomes for more participants if we offered additional encounters for patients who did not respond to the initial intervention.

As such, the purpose of the current pilot study was to pilot test a modified, stepped version of our BRITE-DAY Brief Intervention (BRief Intervention and Treatment for Everyone-Depression Across the Years) for primary care patients with mild to moderate depressive symptoms to evaluate (a) the feasibility and acceptability of the modified intervention and (b) conduct preliminary assessment of clinical outcomes. We conceptualized this pilot study within the NIH Stage Model for behavioral intervention research as Stage I: Intervention Generation/Refinement (Onken et al., 2014). In this model, Stage I activities involve creating, modifying, adapting, or refining an intervention (IA) and pilot testing for feasibility (IB). The authors recommend that this Stage I developmental work involve "routinely and systematically creating and adapting interventions to the intervention delivery context as an integral part of the intervention development process" (p. 6), in order to avoid pitfalls that can occur when efficacious interventions developed in research settings do not translate well in later effectiveness and implementation research or practice. This Stage IB pilot study was conducted with research interventionists, to adapt the materials and test feasibility, acceptability, and preliminary clinical outcomes before proceeding to Stage IB research involving real-world primary care personnel.

We modified the intervention by offering a more flexible number of visits and telephone calls (Table 1). Everyone received at least two telephone calls, and they were offered additional calls and in-person visits every 2 weeks over a period of approximately 3-4 months, based on their residual depressive symptoms and preferences. We also expanded the age limit to encompass all primary care patients, and we extended the follow-up period, assessing outcomes at post-treatment and three months after treatment. We hypothesized that the intervention would be feasible to deliver, that patients would engage in variable numbers of encounters, 
Table 1 Session schedule for stepped BRITE-DAY

\begin{tabular}{|c|c|c|}
\hline \multicolumn{3}{|l|}{ Level 1} \\
\hline Initial call & $15-20 \mathrm{~min}$ & $\begin{array}{l}\text { PHQ-9 } \\
\text { Educate about doing more (psychoeducation handout) } \\
\text { Plan 3-5 activities on activity log }\end{array}$ \\
\hline 2-week call & $10-15 \mathrm{~min}$ & $\begin{array}{l}\text { PHQ-9 } \\
\text { Discuss activities completed, facilitators, barriers } \\
\text { Decide whether to continue }\end{array}$ \\
\hline \multicolumn{3}{|l|}{ Level 2} \\
\hline $\begin{array}{l}\text { Longer call or in-person } \\
\text { clinic visit }\end{array}$ & $30-60 \mathrm{~min}$ & $\begin{array}{l}\text { PHQ-9 } \\
\text { Educate about doing more } \\
\text { Discuss life values } \\
\text { Identify activities from values } \\
\text { Review motivational strategies } \\
\text { Plan 3-5 activities }\end{array}$ \\
\hline 2-week call & $10-15 \mathrm{~min}$ & $\begin{array}{l}\text { PHQ-9 } \\
\text { Discuss activities completed, facilitators, barriers }\end{array}$ \\
\hline 4-week call & $10-15 \mathrm{~min}$ & $\begin{array}{l}\text { PHQ-9 } \\
\text { Discuss activities completed, facilitators, barriers } \\
\text { Decide whether to continue }\end{array}$ \\
\hline \multicolumn{3}{|l|}{ Level 3} \\
\hline 1-6 additional calls & $10-15 \min$ & $\begin{array}{l}\text { PHQ-9 } \\
\text { Discuss activities completed, facilitators, barriers } \\
\text { Decide whether to continue } \\
\text { Termination, relapse prevention, discuss referral } \\
\text { options if indicated }\end{array}$ \\
\hline
\end{tabular}

and that most patients would be satisfied with the intervention. Consistent with our prior pilot study, we also expected to observe improvements on measures of depressive symptoms, anxiety symptoms, disability, and readiness to change at post-treatment that would be maintained three months after the end of treatment, although the design and sample size were not sufficient to formally assess efficacy.

\section{Methods}

\section{Sampling and Recruitment}

Participants were recruited from two General Internal Medicine outpatient clinics of the University of South Florida: one clinic is located on the university campus in a large medical building and the second clinic is part of a collection of outpatient medical clinics in a lower-income, urban residential neighborhood. A research interviewer approached English-speaking patients in private patient rooms and invited them to complete a brief prescreening with the Patient Health Questionnaire-2 (PHQ-2; Kroenke et al., 2003). If they endorsed at least one symptom from the PHQ-2 or indicated other symptoms of depression to the research interviewer (e.g., a patient may have denied depressed mood but stated they were very "stressed"), the interviewer invited them to complete screening with the PHQ-9 (Kroenke et al., 2001) and a brief cognitive screening tool (Callahan et al., 2002). They were eligible for participation if they were aged 18 or older, spoke English, scored 5-14 on the PHQ-9 (indicative of mild-moderate depressive symptoms), and scored 3 or better on the cognitive screening tool (suggesting sufficient cognitive functioning). Figure 1 depicts the screening process, including the numbers of patients approached, pre-screened, screened, and enrolled and the numbers of patients who declined to participate at each phase. The study was approved by the university's Institutional Review Board (Study \#27609), and all participants provided written informed consent. Data were collected from fall 2016 to fall 2017.

\section{BRITE-DAY Brief-Stepped Intervention}

As previously described, the BRITE-DAY Brief Intervention is detailed in a manual, checklist, and readings and worksheets for participants (available from the first author upon request). All activities involve identifying meaningful activities, developing goals to increase their frequency, and tracking when activities are conducted. The patient materials include (a) psychoeducation (overview of how often they would meet with the interventionist, a figure displaying the cycle of doing more and feeling better, features of activities to try, e.g., valued and realistic); (b) worksheet for identifying values and activities across life domains (e.g., family relationships, career, hobbies, and health); (c) readiness ruler and discussion of motivation for change, from our team's brief intervention for alcohol (Schonfeld et al., 2015); and (d) 12-weekly activity $\log$ s to plan and record activities. 
Fig. 1 Participant flow. $P H Q$ 2/9 patient health questionnaire- $2 / 9$

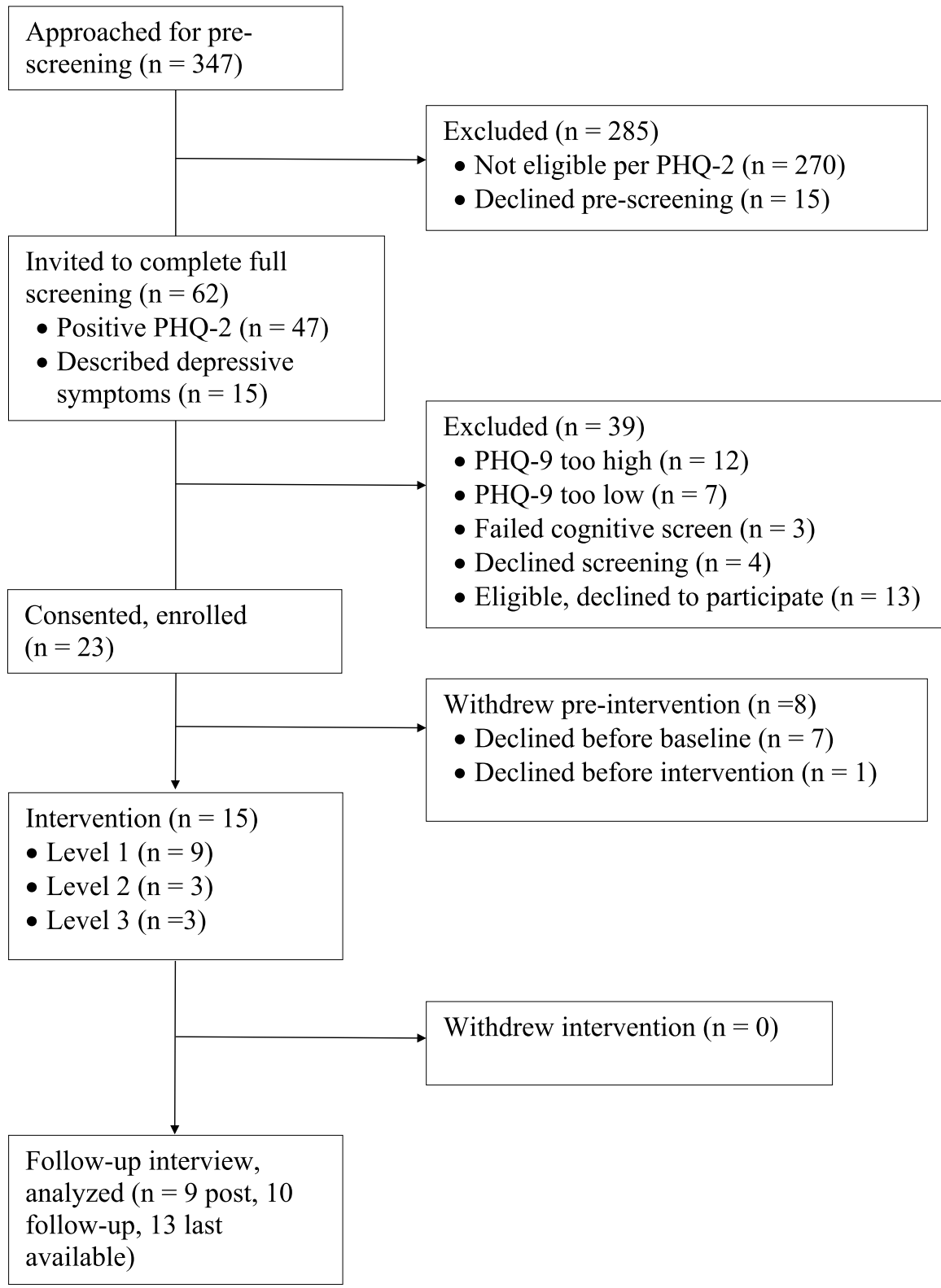

Modifications are described using the Framework for Reporting Adaptations and Modifications-Expanded (FRAME; Stirman et al., 2019). The modifications were planned, before conducting the pilot, by the research team. The goals of the modifications were to improve patient outcomes by providing more encounters for patients who do not respond to the set number of visits allowed in the prior pilot study, as well as to improve feasibility for patients and providers, by offering fewer visits when indicated. The modifications made were to content and contextual, at the level of the individual patient. Content modifications included shortening and condensing some elements in the first level of the intervention and then repeating some elements during the final level of the intervention. Contextual elements included modifying the number of visits and offering patients the choice of whether the visits would occur in-person or by telephone.

For the stepped version evaluated in this pilot study, numbers of visits were based on the participant's PHQ-9 scores over time (Table 1$)$ :

Level 1 ( 2 contacts): All participants received this level of care. Upon enrollment into the study at the clinic, they were provided with the reading materials and worksheets. The interventionist called them shortly thereafter for the first telephone call (15-20 min). Referring to a figure and written notes in the materials, the interventionist briefly explained the rationale (i.e., that as we do more of valued and enjoyable activities, we begin to feel better) and helped the patient to choose and write down 3-5-weekly goals. This initial call was an abbreviated version of the first visit from the prior 
pilot study, shortening the discussion about values, life domains, and more quickly moving to select activities. This initial call was followed by a 10-15-min follow-up telephone calls 2 weeks later to re-administer the PHQ-9 and review progress on completing activities, similar to the follow-up call in the prior version of the protocol.

Level 2 (3 contacts): For participants who continued to exhibit PHQ-9 scores $\geq 5$ at the second Level 1 call, they engaged in a longer session (30-60 min) within 1-2 weeks to more fully review life values and establish weekly goals based on those values. This visit was comparable to the initial visit in the prior version of the protocol, with a more detailed discussion of values, life domains, and activity planning. They could meet the interventionist in person or conduct this session by telephone; all participants elected to meet by telephone. This longer session was followed by two 10-15-min telephone calls 2 weeks and 4 weeks later to re-administer the PHQ-9, review progress, and adjust weekly goals as needed. Thus, the total number of contacts for those completing Level 2 was five.

Level 3 (1-6 contacts): For participants who continued to exhibit PHQ-9 scores $\geq 5$ at the last Level 2 contact, they engaged in 1-6 additional 10-15-min telephone calls every 2 weeks to re-administer the PHQ-9, review progress and adjust weekly goals (in-person visits were offered; all participants who advanced to Level 3 elected to meet by telephone). This level was essentially a repetition of the same follow-up calls from Level 2 . The interventionist and patient ended the intervention when the patient's PHQ-9 fell below 5 or when the patient had reached the maximum number of encounters (up to 6 additional calls; thus the maximum number of contacts for those completing Level 3 was 11).

The first author delivered all intervention activities. This author is a licensed clinical psychologist who has many years of experience delivering and training others in the delivery of brief behavioral interventions. The interventionist completed the checklist for all patient encounters, but no other fidelity assessment was completed for this pilot study. Details regarding numbers of participants who received each level are depicted in Fig. 1.

\section{Measures}

\section{Demographics}

At baseline, participants were asked demographic questions (listed in Table 2).

\section{Feasibility and Acceptability}

Indicators of feasibility and acceptability include domains identified by implementation science frameworks (Proctor et al., 2011; Sekhon et al., 2017).
Table 2 Sample characteristics at baseline $(N=15)$

\begin{tabular}{ll}
\hline Variable & $N(\%)$ or M (SD) \\
\hline Age, M (SD) & $67.07(9.03)$ \\
Range & $52-83$ \\
Gender & \\
Male & $6(40.0)$ \\
Female & $9(60.0)$ \\
Race/ethnicity & \\
White non-Hispanic & $9(60.0)$ \\
Black & $4(26.7)$ \\
White Hispanic & $1(6.7)$ \\
Asian & $1(6.7)$ \\
Education & \\
9th to 11th grade & $1(6.7)$ \\
High school or GED & $3(20.0)$ \\
Some college/trade school & $6(40.0)$ \\
Associates degree & $1(6.7)$ \\
Bachelor's degree & $1(6.7)$ \\
Master's degree or higher & $3(20.0)$ \\
Marital status & \\
Married/live with significant other & $8(53.5)$ \\
Widowed & $2(13.3)$ \\
Divorced/separated & $5(33.3)$ \\
Living arrangement & \\
Live with others & $9(60.0)$ \\
Live alone & $6(40.0)$ \\
Employment status & \\
Employed & $6(40.0)$ \\
Retired & $9(60.0)$ \\
Current psychotropic medication (yes) & $8(53.3)$ \\
Current psychotherapy/counseling (yes) & $1(6.7)$ \\
\hline
\end{tabular}

Numbers represent N(\%) unless otherwise specified

Indicators of feasibility include tracking numbers of individuals approached, screened, eligible, enrolled, intervention encounters in which participants engaged, and research interviews completed (Proctor et al., 2011). Relatedly, refusals to engage in screening or enroll in the study, as well as dropout from the intervention are indicators of acceptability. Also related to acceptability of the intervention (Proctor et al., 2011; Sekhon et al., 2017), several questions were asked about participants' attitudes, experience, satisfaction, and perceived effectiveness of the intervention. Six Likert items assessed overall satisfaction, overall quality, counselor's knowledge and skills, how counselor treats you, how to communicate with counselor, and length of treatment (response options: very satisfied, satisfied, neither, dissatisfied, very dissatisfied). Open-ended items inquired about overall impressions, how the program helped them, changes made because of program, how we could improve program, and whether they would recommend the program to others. 


\section{Clinical Outcomes}

The primary clinical outcome of interest was the PHQ-9 (range 0-27; Kroenke et al., 2001). Other clinical outcomes included readiness to change (University of Rhode Island Change Assessment [URICA] psychotherapy version, range 0-14; McConnaughy et al., 1989), Generalized Anxiety Disorder-7 (GAD-7, range 0-21; Spitzer et al., 2006), and disability (World Health Organization Disability Assessment Schedule. 2.0 [WHODAS 2.0] 12-item version, range 0-48 using the simple scoring method; Andrews et al., 2009; World Health Organization, 2010). All scales have good reliability and validity, and higher scores indicate more of each construct.

\section{Procedures}

Following enrollment, the remaining research and intervention activities were conducted by telephone. Prior to the first intervention phone call, the baseline interview was conducted soon after enrollment in the clinic; it included the demographic questions, URICA, PHQ-9, GAD-7, and WHODAS 2.0 (because the PHQ-9 was repeated at baseline, a participant with scores 5-14 at enrollment could have experienced a change at baseline, moving into the non-depressed or more severely depressed ranges). Intervention activities were then initiated as soon as possible after the baseline, within less than a week unless the patient requested longer or took longer to answer the phone. Followup research interviews were conducted immediately after intervention activities ended (i.e., "post-treatment") and three months after the end of treatment (i.e., "three-month follow-up"); these interviews consisted of the URICA, PHQ9, GAD-7, WHODAS 2.0, and satisfaction questions. All research interviews were conducted by a separate research interviewer, not the interventionist. Participants were not paid for any intervention or research activities; the intervention was delivered to participants at no cost.

\section{Data Analyses}

Data analyses included descriptive statistics for all variables. Frequencies were calculated for numbers of encounters for participants and satisfaction Likert items. To assess pre/ post-changes in clinical outcomes, $t$ tests and Cohen's $d$ for repeated measures $\left(d_{z}=t / V_{n}\right)$ were calculated for PHQ-9, GAD-7, WHODAS 2.0, and URICA, comparing baseline scores to post-treatment, the three-month follow-up, and the last available time point (the last score for each participant-post-treatment or three-month follow-up or, for the PHQ-9 only, the last intervention assessment). Normality of the differences was inspected for the paired t tests; the assumption of normality of the differences was met, based on inspection of plots and Shapiro-Wilk tests. Changes in PHQ-9 categories also were calculated (non-depressed 0-4, mild 5-9, moderate 10-14, moderately severe or higher $>15$; Kroenke et al., 2001). Analyses were conducted using SPSS Version 25.

\section{Results}

As shown in Table 2, participants had an average age of $67.07(\mathrm{SD}=9.03)$, with slightly more females $(\mathrm{N}=9,60 \%)$ than males $(\mathrm{N}=6,40 \%)$. Most participants were white non-Hispanic $(\mathrm{N}=9,60 \%)$ or black $(\mathrm{N}=4,26.7 \%)$. Eleven (73.3\%) had at least some education beyond high school and approximately half were married or living with a partner $(\mathrm{N}=8,53.5 \%)$. Eight $(53.5 \%)$ also were currently taking psychotropic medication, with one $(6.7 \%)$ currently in counseling.

\section{Feasibility and Acceptability}

Figure 1 displays the flowchart of participation, including numbers of individuals approached, screened, enrolled, refused, received the intervention, dropped out, and completed the post-treatment research interviews. As shown in Fig. 1, 23 individuals were enrolled, with 15 (65\%) completing the baseline interview and beginning the intervention. All 15 completed the intervention and had a last available PHQ-9 score, with 13 completing at least one interview after end of treatment (93\%). Comparing those who completed the post-treatment assessment or three-month follow-up assessment to those with missing data, no statistically significant differences were found for demographic variables or baseline scores on outcome measures at either time point, with two exceptions. Compared to those who completed the post-treatment assessment, those with missing data were younger, $M=61.00$ ( $\mathrm{SD}=8.46)$ vs. $M=71.11(\mathrm{SD}=7.20)$, $t(13)=2.49, p=.027$, and had worse WHODAS 2.0 scores at baseline, $M=15.67(\mathrm{SD}=4.55)$ vs. $M=9.11(\mathrm{SD}=5.33)$, $t(13)=-2.47, p=.028$. These differences were not significant for the three-month follow-up assessment.

All 15 participants engaged in Level 1 of BRITE-DAY, six participants completed Level 2, and three completed Level 3. Thus, nine (60\%) participants ended after Level 1 only, three (20\%) participants ended after Level 2, and three (20\%) ended after Level 3.

Regarding acceptability and satisfaction, immediately post-treatment, all participants who completed the assessment $(N=9)$ rated their overall satisfaction as very satisfied $(n=8)$ or satisfied $(n=1)$ and said they would recommend the program to others. All nine also were satisfied or very satisfied with when program activities were offered, quality of services, counselor skills, how counselor treats them, 
counselor communication, and counselor timeliness. Eight were satisfied or very satisfied with how often they met with the counselor and the length of the program, with one participant neither satisfied nor dissatisfied. Eight reported changes they had made (e.g., "be more mindful," "began writing a journal," "more social," "more engaged with friends," "riding my bike").

The satisfaction ratings and comments were very similar at the three-month follow-up ( $n=10$; i.e., all "very satisfied" or "satisfied" with positive comments), except for negative ratings and comments from one participant. He reported "I didn't like it," stated "not enough work put into it on my part" when asked how the program had helped, recommended "more personal contact," and was dissatisfied with how often he had met with the interventionist and the length of the program.

\section{Clinical Outcomes}

Participants demonstrated statistically significant improvements for the PHQ-9, GAD-7, and WHODAS 2.0 from baseline to post-treatment and to the three-month follow-up, as well as for the last available score (Table 3). The effect sizes were large for all comparisons, ranging from 1.30 to 2.25. There was a change in URICA score from baseline to three months with a moderate effect size, but this difference was not significant for post-treatment. Figure 2 displays PHQ-9 scores for baseline, post-treatment, and three-month follow-up by level of intervention, indicating that those who received the higher levels of the interventions had descriptively higher PHQ-9 scores at baseline and all three groups improved, although the numbers were very small (2-3) for levels 2 and 3.

Comparing PHQ-9 categories from baseline to the last available assessment for each participant, two scored moderately severe or higher at baseline; of these, one improved to mild and one improved to non-depressed. Of seven with moderate scores at baseline, all improved to non-depressed. Of five with mild scores at baseline, three improved to nondepressed, one remained mild, and one worsened to moderate (score changed from 8 to 10). The participant with nondepressed score at baseline remained in the non-depressed range (screening PHQ-9 $=5$ for this participant, which met the inclusion criteria for the study; in regular practice, this brief intervention is intended to be initiated same day as screening, in which case this individual would have been offered the intervention; outcome analyses were unchanged after removing this participant, except that URICA was no longer significant at follow-up, $p=.09$ ). In total, at the last available assessment, 12 participants $(80 \%)$ scored in the non-depressed range, two (16.67\%) scored in the mild range, and one $(8.33 \%)$ scored at the border of the moderate range (10).
Table 3 Clinical outcomes over time

\begin{tabular}{|c|c|c|c|c|c|}
\hline & $n$ & $\mathrm{M}(\mathrm{SD})$ & $t(\mathrm{df})$ & $p$ & $d_{z}$ \\
\hline \multicolumn{6}{|l|}{$P H Q-9$} \\
\hline Baseline & 15 & $10.47(4.07)$ & - & - & - \\
\hline Post-treatment & 9 & 3.67 (3.64) & $3.91(8)$ & .004 & 1.30 \\
\hline $\begin{array}{l}\text { Follow-up } \\
\text { (3 months) }\end{array}$ & 10 & $2.20(1.03)$ & $7.13(9)$ & $<.001$ & 2.25 \\
\hline Last available & 15 & $3.40(2.77)$ & $6.44(14)$ & $<.001$ & 1.66 \\
\hline \multicolumn{6}{|l|}{$G A D-7$} \\
\hline Baseline & 15 & $9.40(5.32)$ & - & - & - \\
\hline Post-treatment & 9 & $3.44(4.33)$ & $4.69(8)$ & .002 & 1.56 \\
\hline $\begin{array}{l}\text { Follow-up } \\
\text { (3 months) }\end{array}$ & 9 & $1.11(1.05)$ & $4.08(8)$ & .004 & 1.36 \\
\hline Last available & 13 & $2.54(3.69)$ & $6.09(12)$ & $<.001$ & 1.69 \\
\hline \multicolumn{6}{|l|}{ WHODAS-2.0 } \\
\hline Baseline & 15 & $11.73(5.89)$ & - & - & - \\
\hline Post-treatment & 9 & $5.11(3.62)$ & $4.18(8)$ & .003 & 1.39 \\
\hline $\begin{array}{l}\text { Follow-up } \\
\text { (3 months) }\end{array}$ & 9 & $5.00(3.00)$ & $4.98(8)$ & .001 & 1.66 \\
\hline Last available & 13 & $4.38(3.02)$ & $4.67(12)$ & .001 & 1.30 \\
\hline \multicolumn{6}{|l|}{ URICA } \\
\hline Baseline & 15 & $8.96(1.39)$ & - & - & - \\
\hline Post-treatment & 8 & $8.55(1.45)$ & $-.41(7)$ & .70 & -0.14 \\
\hline $\begin{array}{l}\text { Follow-up } \\
\text { (3 months) }\end{array}$ & 10 & 7.99 (1.69) & $2.38(9)$ & .04 & 0.75 \\
\hline Last available & 13 & $8.36(1.64)$ & $1.39(12)$ & .19 & 0.39 \\
\hline
\end{tabular}

PHQ-9 Patient Health Questionnaire-9; range 0-27, no/minimal $=0-4 ;$ mild $=5-9 ;$ moderate $=10-14 ;$ moderately severe $=15-19$; severe $=20-27$ (Kroenke et al., 2001)

GAD-7 Generalized Anxiety Disorder-7; range $=0-21$, no/minimal $=0-4 ;$ mild $=5-9 ;$ moderate $=10-14 ;$ severe $=15-21 \quad$ (Spitzer et al., 2006)

WHODAS-2.0 World Health Organization Disability Assessment Schedule-2.0 (12-item version; World Health Organization, 2000) range 0-48; means $=3.4$ (ages 55-64), 3.7 (ages 65-74), 5.7 (ages $75-85)$; 50th percentile for ages $55-74=1$, 50th percentile for ages $75-85=3$ (Andrews et al., 2009)

URICA University of Rhode Island Change Assessment (McConnaughy et al., 1989); range $=0-14 ; 0-8$ precontemplation, $8-11$ contemplation, 11-14 preparing into action

Last available $=$ score from last available assessment: 3 -month followup, post-treatment, or last intervention encounter for PHQ-9 only

\section{Discussion}

This pilot study found that the BRITE-DAY-stepped intervention was feasible and acceptable for most patients in a primary care setting, with no patients withdrawing from the intervention after initiation and largely positive ratings and comments regarding acceptability and satisfaction. The intensity of the intervention was fairly low, with most participants receiving the first level of the intervention only ( 2 brief phone calls), suggesting good feasibility for patients as well as potential feasibility for real-world providers. Also 


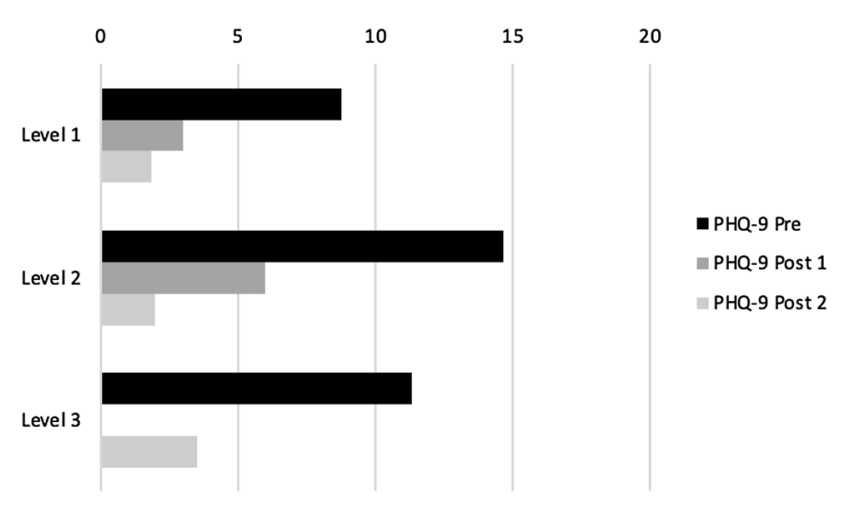

Fig. 2 PHQ-9 scores by level of intervention completed. N's for Pre/ Post-1/Post-2: Level 1=9/7/6, Level $2=3 / 2 / 2$, and Level 3=3/0/2

regarding acceptability, participants reported positive perceived effectiveness of the intervention and reported having made positive behavioral changes aligned with the theorized mechanism of behavioral activation (i.e., increasing valued and enjoyable activities). The exception was one participant who was dissatisfied three months later with the length of the program. Regarding the preliminary evaluation of clinical outcomes, which can also be conceptualized as an indication of acceptability (Sekhon et al., 2017), participants reported improvements in depressive symptoms, anxiety symptoms, and disability compared to baseline, with large effect sizes immediately after treatment and three months later.

The findings from this pilot study are consistent with our prior pilot study, slightly better in fact. In the prior pilot, the observed effect sizes for depressive symptoms, anxiety symptoms, and disability were in the moderate-large range, compared to large effect sizes in the current pilot, and observed depression remission rates were also higher in this study than in our previous pilot ( $80 \%$ vs. $57 \%)$. The one difference was that readiness to change showed less improvement in the current pilot (Gum et al., 2016). Also in the current pilot, participants retained their improvements three months later, whereas the prior study did not assess three-month outcomes.

A noteworthy aspect of these findings is that the clinical outcomes are consistent across both pilot studies, even though the intensity of the intervention was lower in the current pilot study for most participants. In our previous pilot, all participants engaged in one in-person, 90-min visit, followed by three brief telephone calls over the next 4 weeks (Gum et al., 2016). Here, $60 \%$ remitted after one brief telephone-based visit followed by two brief telephone calls over the next 4 weeks (described in Table 1). Three participants $(20 \%)$ engaged in the second level of the intervention, involving three additional phone calls, with a similar total contact time as the original BRITE-DAY protocol (approximately $105-120 \mathrm{~min}$ ). The contact time was more intensive for only three participants (20\%), who engaged in the third level of the intervention (1-6 10-15 min calls). Despite the brevity of the program, participants were satisfied with the quality, time, and counselor's skills, except for one participant at the three-month follow-up. Moreover, all participants elected to speak with the interventionist by telephone instead of coming to the clinic; it may be that most participants liked the convenience of the brief, telephone-based intervention, and may prefer it over more time-intensive, on-site services.

These findings provide support that a very low-intensity, primarily telephone-based behavioral intervention is feasible and acceptable to patients and that this low-intensity intervention may be feasible for delivery as part of a collaborative care management paradigm. The findings from patients' perceived benefits and clinical outcomes also are encouraging and warrant further research to determine whether this brief intervention impacts depressive symptoms. Obvious limitations include the small sample size, pre-post-design with no comparison condition, use of one interventionist without external fidelity ratings, number of enrolled participants who did not begin the intervention, and missing follow-up data for individuals who completed the program. Generalizability for younger primary care patients is also a consideration; although all adult patients $(\geq 18)$ were eligible, the average age of participants in this study was 67 . The research procedures (informed consent, baseline interview) disrupt the intended flow of brief interventions, which could have impacted the numbers of participants who initiated the intervention. Of note, all 15 participants who began the intervention completed it, so engaging participants in the first encounter may be important for retention. Given that younger, more disabled participants were less likely to complete follow-up assessments, more intensive efforts and resources (e.g., stipend, travel to meet participants) are likely needed to engage them in the research interviews after the end of the intervention.

Regarding future research, the NIH Stage Model includes a stage between efficacy (Stage II) and effectiveness research (Stage IV), a Stage III which they refer to as "efficacy testing in the real-world settings" (Onken et al., 2014, p. 7) with real-world providers, in order to ensure that interventions can be feasibly implemented and achieve desired clinical outcomes in the settings for which they were intended (here, primary care). To prepare for such Stage III research, they propose that intervention researchers conduct Stage IB developmental research that explicitly adapts and pilot tests the intervention with the intended providers and delivery context. Thus, we view the next steps for BRITE-DAY as involving further Stage IB developmental research, in which the intervention is delivered by primary care personnel. If that developmental work is successful, then we would proceed to Stage III efficacy research in primary care settings with primary care providers, followed by effectiveness, 
implementation, and/or hybrid research (Curran et al., 2012), ultimately incorporating stepped BRITE-DAY into research on collaborative care programs for depression, in which it would be one of the treatment options that patients could try before medication or could be used to augment medication or other treatment modalities.

It seems possible that an initial behavioral intervention, like this low-intensity stepped BRITE-DAY protocol, could be delivered by medical assistants, nurses, or care managers, with consultative support from behavioral health specialists. It is envisioned that, in regular practice (i.e., without the insertion of research informed consent and baseline research interviews), the initial 10-15-min intervention visit (Level 1) would occur during the same visit as enrollment, as occurring with other brief interventions (e.g., Schonfeld et al., 2015). This practice would likely increase engagement and retention of patients. Engagement and retention also would likely improve if the intervention was being delivered by regular primary care personnel in the context of an existing relationship.

Although data were collected for this study prior to the COVID-19 pandemic, the findings suggest that this primarily telephone-based intervention has applicability during and after the pandemic. Individuals have been more distressed during the COVID-19 pandemic than before (Ammar et al., 2021; Kwong et al., 2021; O'Connor et al., 2021; Proto \& Quintana-Domeque, 2021), and there has been a dramatic increase in telehealth and technology use to meet these needs (Baum et al., 2021; Mansour et al., 2021). The BRITE-DAY intervention relies primarily on telephone-based contact, and the initial visit and follow-ups could be conducted via video telehealth as well. In addition, behavioral activation apps could be incorporated, which have shown positive results thus far (Araya et al., 2021; Huguet et al., 2018).

\section{Conclusion}

In conclusion, results of two pilot studies demonstrate the promise of a very brief behavioral activation intervention for primary care patients with mild-moderate depressive symptoms, with promising evidence of feasibility, acceptability, and benefits. Like another brief intervention our team members have developed and implemented statewide in Florida (Schonfeld et al., 2015), we believe that various primary care personnel (e.g., nurses, medical assistants, primary providers) can learn to implement at least the first components of this intervention, having brief conversations with patients to develop and monitor weekly goals to improve mood and that this intervention could be delivered remotely during and after the COVID-19 pandemic. Thus, the next steps for research regarding this stepped BRITE-DAY intervention are to conduct further pilot testing and efficacy research to adapt it for primary care settings and primary care personnel.

Acknowledgements The authors would like to thank the following for assistance with recruitment and data collection: employees of the University of South Florida General Internal Medicine outpatient clinics and research assistants Kris-Ann Anderson, Brooke Bamford, Alyssa Cooper, and Cassandra Tawadros.

Author Contributions AMG, LS, and LG led the study conception and design. KC contributed to study design. Material preparation, data collection, and data management were performed by AMG and CJ. Data analyses were conducted by AMG, with assistance on qualitative items from CJ. The first draft of the manuscript was written by AMG. All authors commented on previous versions of the manuscript and read and approved the final manuscript.

Funding No funding was received for conducting this study.

Data Availability Upon request from first author.

Code Availability Upon request from first author.

\section{Declarations}

Conflict of interest Amber M. Gum has received compensation from the University of Washington AIMS Center for training behavioral health providers to deliver behavioral interventions in primary care settings (not the intervention under investigation in this pilot study). Claudia Jensen, Lawrence Schonfeld, Kyaien O. Conner, and Lucy Guerra declare that they have no other conflict of interest.

Ethical Approval All procedures performed in this study involving human participants were in accordance with the ethical standards of the institutional research committee (University of South Florida Social and Behavioral IRB, Study \#27609) and with the 1964 Helsinki declaration and its later amendments or comparable ethical standards.

Research Involving Animal Rights This article does not contain any studies with animals performed by any of the authors.

Consent to Participate All participants provided written informed consent.

Consent for Publication Not applicable.

\section{References}

Alexopoulos, G. S., \& Arean, P. (2014). A model for streamlining psychotherapy in the RDoC era: The example of "Engage." Molecular Psychiatry, 19(1), 14-19. https://doi.org/10.1038/mp.2013.150

Alexopoulos, G. S., Raue, P. J., Kiosses, D. N., Seirup, J. K., Banerjee, S., \& Arean, P. A. (2015). Comparing engage with PST in latelife major depression: A preliminary report. American Journal of Geriatric Psychiatry, 23(5), 506-513. https://doi.org/10.1016/j. jagp.2014.06.008

Ammar, A., Trabelsi, K., Brach, M., Chtourou, H., Boukhris, O., Masmoudi, L., \& Ahmed, M. (2021). Effects of home confinement on mental health and lifestyle behaviours during the COVID-19 outbreak: Insights from the ECLB-COVID19 multicentre study. Biology of Sport, 38(1), 9. 
Andrews, G., Kemp, A., Sunderland, M., Von Korff, M., \& Ustun, T. B. (2009). Normative data for the 12 item WHO Disability Assessment Schedule 2.0. PLoS ONE, 4(12), e8343. https://doi.org/10. 1371/journal.pone.0008343

Araya, R., Menezes, P. R., Claro, H. G., Brandt, L. R., Daley, K. L., Quayle, J., \& Miranda, J. J. (2021). Effect of a digital intervention on depressive symptoms in patients with comorbid hypertension or diabetes in Brazil and Peru: Two randomized clinical trials. JAMA, 325(18), 1852-1862. https://doi.org/10.1001/jama.2021. 4348

Areán, P. A., \& Gum, A. M. (2013). Psychologists at the table in health care reform: The case of geropsychology and integrated care. Professional Psychology: Research and Practice, 44(3), 142-149. https://doi.org/10.1037/a0031083

Baum, A., Kaboli, P. J., \& Schwartz, M. D. (2021). Reduced in-person and increased telehealth outpatient visits during the COVID-19 pandemic. Annals of Internal Medicine. https://doi.org/10.7326/ M20-3026

Bower, P., Gilbody, S., Richards, D., Fletcher, J., Sutton, A., Bower, P., $\&$ Sutton, A. (2006). Collaborative care for depression in primary care. Making sense of a complex intervention: Systematic review and meta-regression. British Journal of Psychiatry, 189, 484-493.

Callahan, C. M., Unverzagt, F. W., Hui, S. L., Perkins, A. J., \& Hendrie, H. C. (2002). Six item screener to identify cognitive impairment among potential subjects for clinical research. Medical Care, 40, $771-781$.

Chartier, I. S., \& Provencher, M. D. (2013). Behavioural activation for depression: Efficacy, effectiveness and dissemination. Journal of Affective Disorders, 145(3), 292-299. https://doi.org/10.1016/J. Jad.2012.07.023

Community Preventive Services Task Force. (2012). Recommendation from the community preventive services task force for use of collaborative care for the management of depressive disorders. American Journal of Preventive Medicine, 42(5), 521-524. https://doi.org/10.1016/j.amepre.2012.01.010

Cross, W. F., Pisani, A. R., Schmeelk-Cone, K., Xia, Y., Tu, X., McMahon, M., \& Gould, M. S. (2014). Measuring trainer fidelity in the transfer of suicide prevention training. Crisis, 35(3), 202-212. https://doi.org/10.1027/0227-5910/a000253

Curran, G. M., Bauer, M., Mittman, B., Pyne, J. M., \& Stetler, C. (2012). Effectiveness-implementation hybrid designs: Combining elements of clinical effectiveness and implementation research to enhance public health impact. Medical Care, 50(3), 217.

Dimidjian, S., Barrera, M., Jr., Martell, C., Munoz, R. F., \& Lewinsohn, P. M. (2011). The origins and current status of behavioral activation treatments for depression. Annual Review of Clinical Psychology, 7, 1-38. https://doi.org/10.1146/annurev-clinpsy032210-10453521275642

Funderburk, J. S., Pigeon, W. R., Shepardson, R. L., \& Maisto, S. A. (2020). Brief behavioral activation intervention for depressive symptoms: Patient satisfaction, acceptability, engagement, and treatment response. Psychological Services, 17(4), 443.

Funderburk, J. S., Pigeon, W. R., Shepardson, R. L., Wade, M., Acker, J., Fivecoat, H., \& Maisto, S. A. (2021). Treating depressive symptoms among veterans in primary care: A multi-site RCT of brief behavioral activation. Journal of Affective Disorders, 283, $11-19$.

Gawrysiak, M., Nicholas, C., \& Hopko, D. R. (2009). Behavioral activation for moderately depressed university students: Randomized controlled trial. Journal of Counseling Psychology, 56(3), 468.

Gensichen, J., von Korff, M., Peitz, M., Muth, C., Beyer, M., Güthlin, C., \& Gerlach, F. M. (2009). Case management for depression by health care assistants in small primary care practices: A cluster randomized trial. Annals of Internal Medicine, 151(6), 369-378.

Gros, D. F., \& Haren, W. B. (2011). Open trial of brief behavioral activation psychotherapy for depression in an integrated veterans affairs primary care setting. Primary Care Companion for CNS Disorders, 13(4), PCC.11m01136. https://doi.org/10.4088/PCC. $11 \mathrm{~m} 01136$

Gum, A. M., Arean, P. A., Hunkeler, E., Tang, L., Katon, W., Hitchcock, P., \& Unutzer, J. (2006). Depression treatment preferences in older primary care patients. The Gerontologist, 46(1), 14-22.

Gum, A. M., Schonfeld, L., Tyler, S., Fishleder, S., \& Guerra, L. (2016). One-visit behavioral intervention for older primary care patients with mild-moderate depressive symptoms: A pilot study. Southern Medical Journal, 109, 442-447. https://doi.org/10. 14423/SMJ.0000000000000497

Hooker, S. A., Slattengren, A. H., Boyle, L., \& Sherman, M. D. (2020). Values-based behavioral activation for chronic pain in primary care: A pilot study. Journal of Clinical Psychology in Medical Settings, 27(4), 633-642.

Hopko, D. R., Bell, J. L., Armento, M. E. A., Hunt, M. K., \& Lejuez, C. W. (2005). Behavior therapy for depressed cancer patients in primary care. Psychotherapy: Theory, Research, Practice, Training, 42(2), 236-243. https://doi.org/10.1037/0033-3204.42.2.236

Huguet, A., Miller, A., Kisely, S., Rao, S., Saadat, N., \& McGrath, P. J. (2018). A systematic review and meta-analysis on the efficacy of Internet-delivered behavioral activation. Journal of Affective Disorders, 235, 27-38.

IOM (Institute of Medicine). (2015). Psychosocial interventions for mental and substance use disorders: A framework for establishing evidence-based standards. The National Academies Press.

Katon, W. J., \& Unützer, J. (2013). Health reform and the Affordable Care Act: the importance of mental health treatment to achieving the triple aim. Journal of Psychosomatic Research, 74(6).

Katon, W. J., Unützer, J., Wells, K., \& Jones, L. (2010). Collaborative depression care: History, evolution and ways to enhance dissemination and sustainability. General Hospital Psychiatry, 32(5), 456-464.

Kroenke, K., Spitzer, R. L., \& Williams, J. B. W. (2001). The PHQ-9: Validity of a brief depression severity measure. Journal of General Internal Medicine, 16, 606-613.

Kroenke, K., Spitzer, R. L., \& Williams, J. B. W. (2003). The Patient Health Questionnaire-2: Validity of a two-item depression screener. Medical Care, 41, 1284-1292.

Kwong, A. S., Pearson, R. M., Adams, M. J., Northstone, K., Tilling, K., Smith, D., \& Zammit, S. (2021). Mental health before and during the COVID-19 pandemic in two longitudinal UK population cohorts. The British Journal of Psychiatry, 218(6), 334-343.

Mansour, O., Tajanlangit, M., Heyward, J., Mojtabai, R., \& Alexander, G. C. (2021). Telemedicine and office-based care for behavioral and psychiatric conditions during the COVID-19 pandemic in the United States. Annals of Internal Medicine. https://doi.org/ 10.7326/M20-6243

McConnaughy, E. A., DiClemente, C. C., Prochaska, J. O., \& Velicer, W. F. (1989). Stages of change in psychotherapy: A follow-up report. Psychotherapy, 26(4), 494-503.

Miller, B. F., Ross, K. M., Davis, M. M., Melek, S. P., Kathol, R., \& Gordon, P. (2017). Payment reform in the patient-centered medical home: Enabling and sustaining integrated behavioral health care. American Psychologist, 72(1), 55-68.

O'Connor, R. C., Wetherall, K., Cleare, S., McClelland, H., Melson, A. J., Niedzwiedz, C. L., \& Scowcroft, E. (2021). Mental health and well-being during the COVID-19 pandemic: Longitudinal analyses of adults in the UK COVID-19 Mental Health \& Wellbeing study. The British Journal of Psychiatry, 218(6), 326-333.

Onken, L. S., Carroll, K. M., Shoham, V., Cuthbert, B. N., \& Riddle, M. (2014). Reenvisioning clinical science: Unifying the discipline to improve the public health. Clinical Psychological Science, 2(1), $22-34$.

Press, M. J., Howe, R., Schoenbaum, M., Cavanaugh, S., Marshall, A., Baldwin, L., \& Conway, P. H. (2017). Medicare payment for 
behavioral health integration. New England Journal of Medicine, 376(5), 405-407.

Proctor, E., Silmere, H., Raghavan, R., Hovmand, P., Aarons, G., Bunger, A., \& Hensley, M. (2011). Outcomes for implementation research: Conceptual distinctions, measurement challenges, and research agenda. Administration and Policy in Mental Health and Mental Health Services Research, 38(2), 65-76.

Proto, E., \& Quintana-Domeque, C. (2021). COVID-19 and mental health deterioration by ethnicity and gender in the UK. PLoS ONE, 16(1), e0244419.

Reynolds, C. F., III., Cuijpers, P., Patel, V., Cohen, A., Dias, A., Chowdhary, N., \& Albert, S. M. (2012). Early intervention to reduce the global health and economic burden of major depression in older adults. Annual Review of Public Health, 33, 123-135. https://doi.org/10.1146/annurev-publhealth-031811-124544

Richards, D. A., Hill, J. J., Gask, L., Lovell, K., Chew-Graham, C., Bower, P., \& Bland, J. M. (2013). Clinical effectiveness of collaborative care for depression in UK primary care (CADET): Cluster randomised controlled trial. The BMJ, 347, f4913.

Schonfeld, L., Hazlett, R. W., Hedgecock, D. K., Duchene, D. M., Burns, L. V., \& Gum, A. M. (2015). Screening, brief intervention, and referral to treatment for older adults with substance misuse. American Journal of Public Health, 105, 205-211. https://doi.org/ 10.2105/AJPH.2013.301859

Sekhon, M., Cartwright, M., \& Francis, J. J. (2017). Acceptability of healthcare interventions: An overview of reviews and development of a theoretical framework. BMC Health Services Research, $17(1), 1-13$.

Spitzer, R. L., Kroenke, K., Williams, J. B. W., \& Lowe, B. (2006). A brief measure for assessing generalized anxiety disorder: The GAD-7. Archives of Internal Medicine, 166(10), 1092-1097. https://doi.org/10.1001/archinte.166.10.1092

Stein, A. T., Carl, E., Cuijpers, P., Karyotaki, E., \& Smits, J. A. (2021). Looking beyond depression: A meta-analysis of the effect of behavioral activation on depression, anxiety, and activation. Psychological Medicine, 51(9), 1491-1504.

Stirman, S. W., Baumann, A. A., \& Miller, C. J. (2019). The FRAME: An expanded framework for reporting adaptations and modifications to evidence-based interventions. Implementation Science, 14(1), 1-10.

Sturmey, P. (2009). Behavioral activation is an evidence-based treatment for depression. Behavior Modification, 33(6), 818-829. https://doi.org/10.1177/014544550935009419933444

Trombello, J. M., South, C., Cecil, A., Sanchez, K. E., Sanchez, A. C., Eidelman, S. L., \& Trivedi, M. H. (2017). Efficacy of a Behavioral Activation teletherapy intervention to treat depression and anxiety in primary care: VitalSign6 program. The Primary Care Companion for CNS Disorders, 19(5), 17m02146. https://doi.org/ 10.4088/PCC.17m02146

Unützer, J., Katon, W., Callahan, C. M., Williams, J. W., Jr., Hunkeler, E., Harpole, L., Hoffing, M., Della Penna, R. D., Noël, P. H., Lin, E. H., Areán, P. A., Hegel, M. T., Tang, L., Belin, T. R., Oishi, S., Langston, C., \& IMPACT Investigators. Improving Mood-Promoting Access to Collaborative Treatment. (2002). Collaborative care management of late-life depression in the primary care setting: A randomized controlled trial. JAMA, 288(22), 2836-2845. https:// doi.org/10.1001/jama.288.22.2836

Woltmann, E., Grogan-Kaylor, A., Perron, B., Georges, H., Kilbourne, A. M., \& Bauer, M. S. (2012). Comparative effectiveness of collaborative chronic care models for mental health conditions across primary, specialty, and behavioral health care settings: Systematic review and meta-analysis. American Journal of Psychiatry, 169(8), 790-804. https://doi.org/10.1176/appi.ajp.2012.11111616

World Health Organization. (2000). World Health Organization Disability Assessment Schedule (WHODAS II). WHO.

World Health Organization. (2010). World Health Organization Disability Assessment Schedule-WHODAS 2.0. WHO.

Zivin, K., O'Malley, A., Bigby, J., Brown, J., \& Rich, E. (2016). Behavioral health integration in primary care: A review and implications for payment reform. Mathematica Policy Research.

Publisher's Note Springer Nature remains neutral with regard to jurisdictional claims in published maps and institutional affiliations. 